American Journal of Applied Sciences 4 (5): 333-338, 2007

ISSN 1546-9239

(C) 2007 Science Publications

\title{
A Novel Multicast Routing Protocol for Mobile Ad Hoc Networks
}

\author{
${ }^{1}$ Zeyad M. Alfawaer, GuiWei Hua, and ${ }^{2}$ Noraziah Ahmed \\ ${ }^{1}$ School of Information Sciences and Engineering Central South University, \\ Changsha 410083-Hunan-P.R.China \\ ${ }^{2}$ University Malaysia Pahang, Faculty of Computer System and Software Engineering, \\ Locked Bag 12, 25000 Kuantan, Pahang, Malaysia.
}

\begin{abstract}
In mobile ad hoc networks, how to achieve the multicast communication is a challenging task due to the fact that the topology may change frequently and communication links may be broken because of users' mobility. We introduced MANHSI (Multicast for Ad hoe Network with hybrid Swarm Intelligence) protocol, which relies on a swarm intelligence based optimization technique to learn and discover efficient multicast connectivity. The proposed protocol instances that it can quickly and efficiently establish initial multicast connectivity and/or improved the resulting connectivity via different optimization techniques. Using a simulation approach, we investigated performance of the proposed algorithm through a comparison with an algorithm previously proposed in the literature. Based on the numerical results, we demonstrate that our proposed algorithm performs well.
\end{abstract}

Key word: Mobile ad hoc networks, multicast algorithm, hybrid Swarm Intelligence, optimization techniques.

\section{INTRODUCTION}

In mobile wireless ad hoc networks attract increasing interest in many application domains since they may be the only solution in situations, where resources such as energy and bandwidth are scarce, it is preferred that networking protocols are resource aware. From the aspect of multicast routing, multicast protocols should be able to establish efficient connectivity among group members with an acceptable level of overhead. One way to achieve this is to find a subset of nodes that can be used to connect all the group members together while yielding the minimum total "cost." However, finding such a minimum-cost subset is similar to the Steiner tree problem [1,2], which is known to be NPhard. Although several heuristics have been proposed, they often rely on global knowledge of network topology to perform the calculation.

In traditional static IP networks, the goal of multicast routing is to find a tree of links connecting all routers that belong to a certain multicast group. However, IP multicast protocols [3-5] are inappropriate for ad hoc networks because multicast trees could easily break due to dynamic topologies [6]. Many multicast protocols for ad hoc networks have been proposed.

Some protocols still rely on constructing a tree spanning all group members $[7,8]$, which is not robust enough when the network becomes more dynamic with less reliable wireless links. In contrast, many proposed protocols have data packets transmitted into more than one link, and allow packets to be received on links that are not branches of a multicast tree. These protocols fall into a category of mesh-based protocols in that group connectivity is formed as a mesh rather than a tree to increase robustness at the price of adding more redundancy in data transmission. Flooding, where data packets are forwarded to and received from all links, is also considered a mesh protocol since the mesh is in fact the entire network topology. In highly dynamic, highly mobile ad hoc networks, a flooding approach is a better alternative to multicast routing due to its minimal state maintained and high reliability [9].

As the extreme, flooding provides the most robust, but inefficient mechanism since a multicast packet will be forwarded to every node (as long as the network is not partitioned), while tree-based approach offers efficiency but is not robust enough to be used in highly dynamic environments. Furthermore, routing based on a connected dominating set [10-12] can also increase the overall efficiency since the search space is reduced to only nodes in the dominating set during route discovery and maintenance processes.

This paper develops a novel multicast routing protocol for mobile ad hoc networks that adopts swarm intelligence to reduce the number of nodes used to

Corresponding Author: Zeyad M. Alfawaer, School of Information Sciences and Engineering Central South University, Changsha 410083-Hunan-P.R.Ch 
establish multicast connectivity, which allows multicast connections of lower total costs to be learned over time.

Multicast Techniques for Mobile Ad hoc Networks: To provide multicast routing over mobile ad hoc networks, the challenge is to effectively handle frequent topology changes caused by node mobility/failure and link disruption due to interference and jamming. A number of multicast techniques have been proposed to address this issue. These protocols are ranging from a simple flooding scheme to state-based tree or mesh structures, as well as hierarchical and hybrid approaches. Based on their operations, there exist different taxonomy schemes to classify these ad hoc multicast routing protocols, including connectivity among group members (tree-based vs mesh-based), route acquisition schemes (proactive vs reactive), connectivity initialization (sender-initiated vs receiverinitiated) , dependency on unicast routing, and forwarding state maintenance schemes (source-based vs group-shared). Most ad hoe multicast protocols propose different approaches based on different assumptions about the environments such as mobility speeds. Several adaptive multicast protocols have been proposed to incorporate different behaviors within the same protocol. Fig.1 categorizes the aforementioned protocols based on the methodologies of maintaining connectivity among multicast group members.

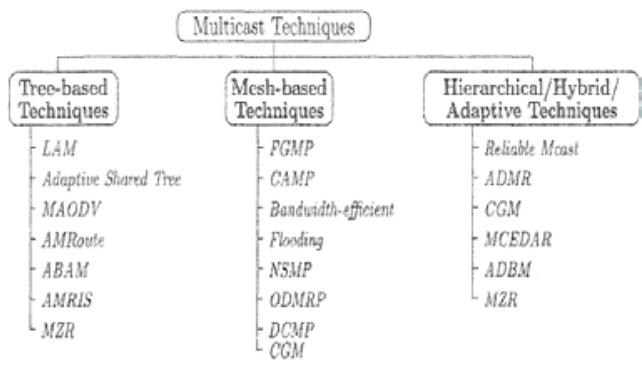

Fig. 1: Classification of multicast protocols for ad hoc networks based on member connectivity

Multicast with Hybrid Swarm Intelligence: A novel multicast routing protocol for mobile ad hoc networks that adopts swarm intelligence to reduce the number of nodes used to establish multicast connectivity, which allows multicast connections of lower total costs to be learned over time.

Swarm intelligence refers to complex behaviors that arise from simple interactions among individuals, such as ants, but often achieves global optimization objectives.

Similarly, MANHSI utilizes small control packets equivalent to ants in the physical world. These packets, traveling like biological ants, deposit control information at nodes they visit similar to the way ants laying pheromone trails. This information, in turn, affects the behavior of other ant packets. With this form of indirect communication, the deployment of ant-like packets resembles an adaptive distributed control system that evolves itself to a more efficient state, accommodating the current condition of the environment.

For each multicast group, MANHSI determines a set of intermediate nodes, forming forwarding set, those connects group members together and are shared among group senders. By adopting a core-based approach, the forwarding set is initially formed by nodes that are on the shortest paths between the core and the other group members, where the core may be one of the group members or senders.

In addition, during the lifetime of the multicast session (i.e., when there is at least one active sender), the forwarding set will evolve, by means of swarm intelligence, over time into states that yield lower cost, which is expressed in terms of total cost of all the nodes in the forwarding set. This evolving, including exploring and learning, mechanism differentiates MAKSI from other existing ad hoc multicast routing protocols. Since a node's cost is abstract and may be defined to represent different metrics, MANHSI can be applied to many variations of multicast routing problems for ad hoc networks such as load balancing, secure routing, and energy conservation.

Mobile Ad hoc network with hybrid swarm intelligence (MANHSI): MAYSI is an on-demand multicast routing protocol that creates a multicast connection among group members by determining a set of intermediate nodes that serve as forwarding nodes. This set, called a forwarding set, is shared among all the senders of the group. The protocol exploits a core-based technique where each member joins the group via the core node to establish a connection with the other group members. Unlike the core-based tree (CBT) protocol [3], however, the core of each group is not statically assigned to a particular node in the network and is not known in advance by the members. Instead, the first member who becomes an active source (i.e., starts sending data to the group) takes the role of the core and announces its existence to the others by floodirrg the network with a COREA ANNONCE packet. Each member node then relies on this announcement to reactively establish initial connectivity by sending a JOIN Request ck to the core via the reverse path. Nodes who receive a JOINR Request dressed to themselves become forwarding nodes of the group and are 
responsible for accepting and rebroadcast nonduplicated data packets, regardless of which node the packets were received from. Therefore, MANHSI does not rely on any unicast routing protocol.

To maintain connectivity and allow new members to join, the core floods CORE ANNOUNC periodically as long as there are more data to be sent. As a result, these forwarding nodes form a mesh structure that connects the group members together, while the core serves as a focal point for forwarding set creation and maintenance. Since this process is performed only when there is an active source sending data to the group, we do not waste valuable network bandwidth to unnecessarily maintain group connectivity in such dynamic environments.

Similar to other core-based protocols, this process creates a forwarding set consisting of all the intermediate nodes on the paths on which CORE ANNOUNCES are accepted and forwarded from the core to the other members, which are often shortest paths, as illustrated in Fig.2(a). However, group connectivity can be made more efficient by having node $A$ choose another path that is partially shared by node $B$ to reduce the size of the forwarding set, as shown in Fig.2(b), which lowers the total cost of forwarding data packets. Note that the cost is considered on a per-node basis, not per-link, due to the fact that wireless communication is broadcast in nature (i.e., a single data packet broadcast by a node is expected to arrive at all of its immediate neighbors in one transmission). In general, the cost of the forwarding set does not always reflect the number of nodes in the set. Instead, the cost associated with each node can represent different measurements, depending on the desired properties of the forwarding set. For instance, if we aim to reduce the number of nodes in the forwarding set for efficient data forwarding, the cost associated with each node could be one. Table 1 lists a few more examples of what node cost would represent when MANHSI is applied to other variations of the multicast routing problem in wireless ad hoc networks.

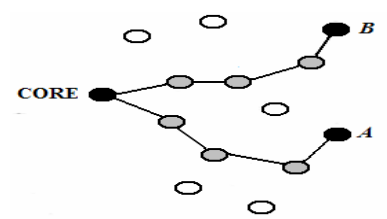

(a)

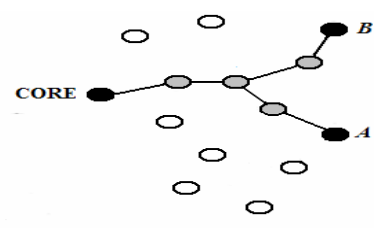

(b)
Fig.2: Examples of multicast connectivity among three group members: (a) a forwarding set of six nodes formed by shortest paths from the core to the other two members, and (b) another forwarding set when node A partially shares the same path to the core with node $B$, which results in more efficient data packet forwarding
Table 1: A few variations of the multicast routing problem and how each node would compute its cost in MANHSI

\begin{tabular}{ll}
\hline Problem & $\begin{array}{l}\text { Cost calculation per node } \\
\text { Load balancing }\end{array}$ \\
size
\end{tabular}

We adopt the swarm intelligence metaphor to allow nodes to learn a better multicast connection that yields a lower (total) forwarding cost. Each member who is not the core periodically deploys a small packet, called a FORWARAD ANT, that opportunistically explores different, and hopefully better paths toward the core. This exploring process is illustrated in Fig.3. If a FORWARAD ANT arrives at a node who is currently serving as a forwarding node for the group (node $D$ in this case), the ant turns itself into a BACKWARD ANT and travels back to its originator via the reverse path. When the BACKWARD ANT arrives at each intermediate node, it estimates the cost of having the node it is currently at join the forwarding set via the forwarding node it previously found. The computed cost, as well as a pheromone amount that is inversely proportional to the cost, are updated on the node's local data structure. These pheromone amounts are then used by subsequent FORWARD ANTS that arrive at this node to make a decision which node they will travel to next, similar to how pheromone is used by biological ants. Let us consider the same example shown in Fig.3, when the BACKWARD ANT leaves node $D$ and arrives at node $C$, the cost of having node $C$ join the forwarding set via node $D$ is zero since node $D$ is already a forwarding node and is directly connected to node $\mathrm{C}$.

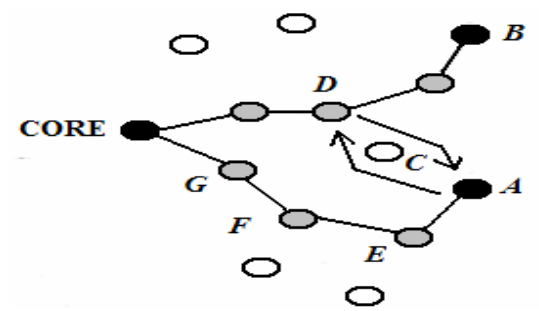

Fig.3: Behavior of forward and backward ants: (1) a FORWARD AN $\mathrm{T}$ deployed from the member A choosing node $\mathrm{C}$ as the next hop and encountering a forwarding node $\mathrm{D}$, and (2) at node $\mathrm{D}$, the FORWARD ANT becoming a BACKWARD ANT and following the reverse path back to node $A$ while depositing pheromone along the way 
When the ant comes back to node $\mathrm{A}$, the cost of having node A join the forwarding set via node $D$ is the same as the cost associated with node $C$ because node $C$ would be required to become a forwarding node to allow node $A$ to join the group via node $D$. If node $A$ sees that the pheromone amount on the link to node $\mathrm{C}$ becomes the highest among links to node neighboring nodes, it will switch to join the group via node $\mathrm{C}$ by sending a JOIN REQUEST to node $C$. Consequently, node $C$ will become a forwarding node, while nodes $E$, $F$, and $G$ will remove themselves from the forwarding set (since they no longer hear requests from node A), which is similar to the connectivity shown in Fig. 2(b).

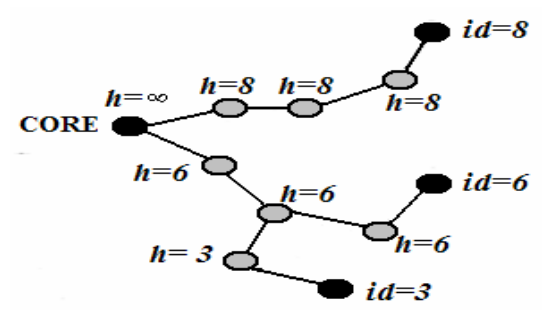

Fig. 4: An example illustrating how heights are assigned to forwarding node $\mathrm{s}$ used by the members with IDS 3, 6 and 8

To prevent the race condition where members attempt to establish group connectivity via one another's forwarding path and nobody remains connected to the core, each forwarding node is associated with a height which is identical to the highest ID of the nodes that use it to connect to the core. En addition, the core has its height set, to infinity. Fig.4 shows an example illustrating how heights are assigned to forwarding nodes. A FORWARD Ants must stop and turn into a BACKWARD ANT to only when it encounters a forwarding node whose height is higher than the ID of the member who originated the ant. That means a member is allowed to connect to the core via an existing path that belongs to another member with a higher ID, but not vice versa, to assure that the core, whose height is always the highest, will eventually be connected to all the other members.

By following these simple rules, a majority of FORWARD ANTS from each member will choose a path that connects to an existing forwarding node with a smaller total path cost. Nodes on this path are then used to forward multicast data packets, resulting in a lower data forwarding cost. This exploring and learning mechanism enables MANHSI to learn a better forwarding set for each group, depending on how node cost is defined, as well as differentiates MAKSI from other existing ad hoe multicast routing protocols. Note that, by doing so, MANHSI attempts to evolve multicast connectivity into states that yield lower cost. It, however, does not guarantee that minimum-cost connectivity can be achieved.

\section{RESULTS AND DISCUSSION}

To study the characteristics and evaluate the performance of MANHSI, we have conducted simulation experiments using the Qual Net simulator. Ten random networks were generated with 50 nodes uniformly distributed over a terrain of size $1000 \times 1000$ $\mathrm{m}^{2}$. Each node was equipped with a radio transceiver which was capable of transmitting signals up to approximately 250 meters over a 2 Mbps wireless channel, using the two-ray path loss model without fading. We used IEEE 802.11DCF as the MAC layer protocol, and IP as the network layer. Since MANHSI does not rely on any unicast routing protocol, no other routing protocols were employed. For each network, a multicast group of 5 members was setup, where each member generated a constant bit rate (CBR) traffic at 2 packets/second to the group for 20 minutes.

Our first set of experiments was setup without mobility to study how MANHSI maintains forwarding sets in static environments. For comparison purposes, we used two base line protocols: FLOOD and CORE, as references. FLOOD is a simple flooding protocol where a data packet is rebroadcast by every node in the network, and CORE is a generic core-based protocol that operates exactly like MANHSI, but without ants deployed, where CORE ANNOUNCE are periodically flooded as usual.

The cost of each node was set to one, which implies that MANHSI would attempt to reduce the size of the forwarding set.

We first look at the average size of forwarding sets maintained by CORE and MANHSI over time for the ten sample networks, as shown in Figure 5. Due to random delays added to avoid packet collisions when broadcasting, the dissemination pattern of a COREA NNOUNCE is unpredictable when it is Hooded, which causes forwarding set to be formed differently after each announcement. Consequently, the average size of forwarding sets keeps changing from time to time in CORE. In contrast, forwarding sets maintained by MANHSI start of at around the same size as that of CORE but keep reducing in size during the first 200 seconds. Their size then becomes stable and stays low most of the time as each member or forwarding node tends to join the group via a low-cost path (i.e., small hop count in this case), whose existence was recently confirmed by BACKWARDA NTS. Although another 
COREA NNOUNC may arrive at a member from a different node, the member will not send a JOIN REQUEST to this new node as long as the current joining cost is low and the pheromone intensity on the link it currently uses to join the group is

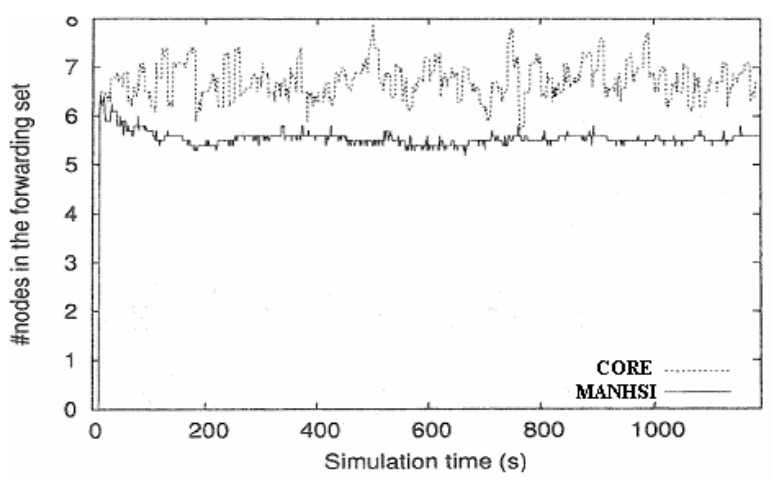

Fig. 5: Average size of the forwarding set as a function of time for CORE and MANHS1

Table 2 Average size of the forwarding set formed in MANHSI, CORE, and FLOOD for each network high.

\begin{tabular}{cccc}
\hline Network & Average Size \\
& MANHSI & CORE & FLOOD \\
\hline 1 & 7.9 & 9.5 & 50.0 \\
2 & 3.0 & 3.7 & 50.0 \\
3 & 4.0 & 5.0 & 50.0 \\
4 & 4.5 & 4.7 & 50.0 \\
5 & 6.5 & 8.5 & 50.0 \\
6 & 4.5 & 6.3 & 50.0 \\
7 & 6.9 & 7.8 & 50.0 \\
8 & 7.5 & 7.5 & 50.0 \\
9 & 5.2 & 7.7 & 50.0 \\
10 & 4.0 & 7.0 & 50.0 \\
Average & 5.3 & 6.7 & 50.0 \\
\hline
\end{tabular}

Table 2 summarizes the sizes, averaged over the entire simulation time, of the forwarding sets maintained by MANHSI, CORE, and FLOOD on each simulated network. (FLOOD does not really maintain a forwarding set, but the set consists of every node in the network.) The results show that in all cases, except one, MANHSI yields forwarding sets that are approximately $15 \%-20 \%$ smaller than those of CORE, and much smaller than FLOOD. Since the size of the forwarding set indicates how nearly nodes are involved to relay a data packet from one member to the others, this demonstrates the efficiency of MANST in terms of data forwarding.

Fig. 6 presents packet delivery ratio of the protocols at different mobility speeds. MANHSI without the mobility-adaptive mechanism, denoted by MANHSI Basic, shows significant performance degradation as mobility increases due to the fact that the forwarding set lacks redundant paths when each member and forwarding node always requests only one of its neighbor to be part of the forwarding set. However, when the mobility-adaptive mechanism is enabled, as denoted by MANHSI-Mobile, its results are comparable with FLOOD. Although the delivery ratio is a bit lower than that of the other two protocols, more than $90 \%$ of data packets can be delivered at every mobility speed.

In terms of efficiency, both MANHSI-Basic and MANHSI-Mobile give significantly better performance than FLOOD at low mobility in both channel access and bandwidth utilization aspects, as shown in Fig.7 and Fig.8, respectively.

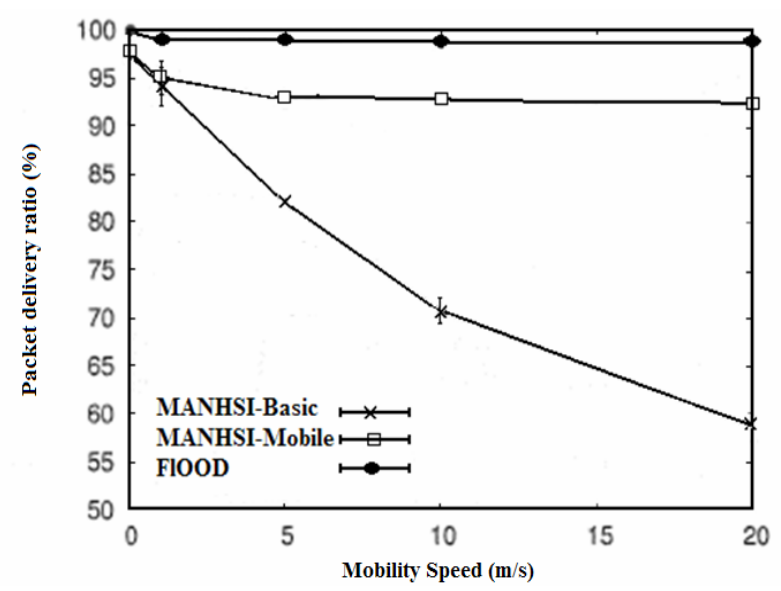

Fig. 6: Packet delivery ratio as a function of mobility speed

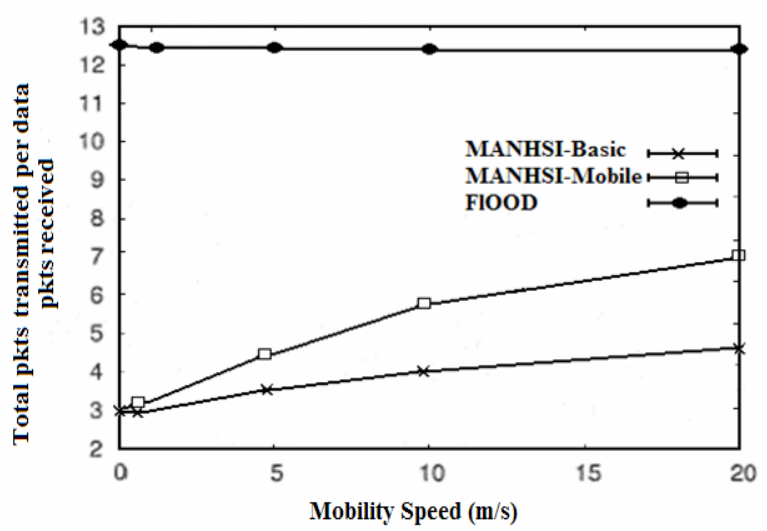

Fig.7: Total packets transmitted per data packet received at the destinations as a function of mobility speed 


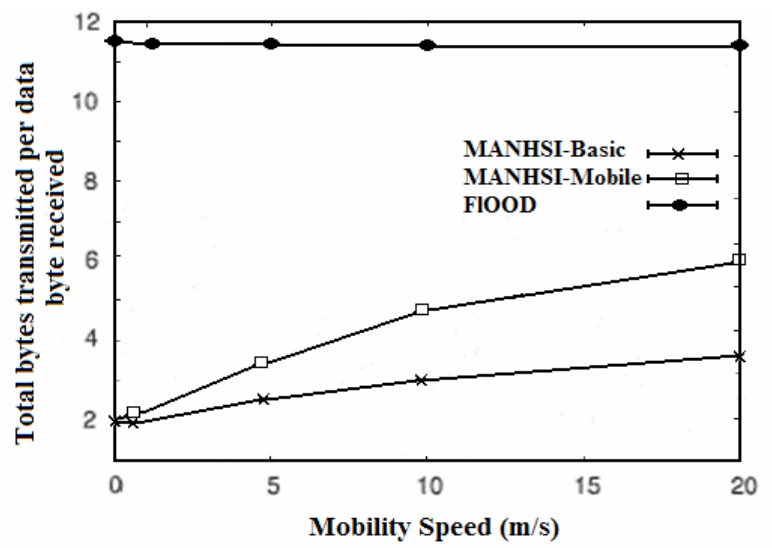

Fig.8: Total bytes transmitted per data byte received at the destinations as a function of mobility speed

\section{CONCLUSION}

Inspired by swarm intelligence, we have introduced an alternative approach to solving the multicast rotating problem in mobile ad hoe networks. The protocol, called MANHSI (Multicast for Ad hoe Networks with Swarm Intelligence), is an on demand multicast routing protocol that creates a multicast mesh shared by all the members within each group. The protocol uses a corebased scheme, where each member initiates a request to the core node to establish multicast connectivity with other members. Intermediate nodes who receive such a request become forwarding nodes that are used to relay data packets from one member to the others. Unlike other core-based protocols, MANHSI does not always rely on the shortest paths between the core and the members to establish group connectivity. Instead, each member who is not the core periodically deploys a small packet that behaves like an ant to opportunistically explore different paths. This exploring mechanism enables the protocol to discover paths that comprise a better set of forwarding nodes yielding a lower total cost of data forwarding, where the "cost" of forwarding (nodes) can be defined in terms of different application specific performance metrics. MANHSI also incorporates a mobility-adaptive mechanism that allows the protocol to remain effective as mobility increases. The simulation results have shown that MANHSI performs both effectively and efficiently in static or low-mobility environments, yet still effectively in highly dynamic environments.

\section{REFERENCES}

1. Madaba Barath-Mumar and Jeffrey M. Jaffe, 1983. Routing to Multiple Destinations in Computer Networks. IEEE Transactions on Communications, 31 (3).

2. H. Eim and c. Kim, 2000. Multicast Tree Construction and Flooding in Wireless Ad Hoc Networks. In AC-Ad International Workshop on Modeling, Analysis and Simulation of Wireless and Mobile Systems (MSWIM).

3. Tony Ballardie, Paul Rancis, and Jon Crowcroft 1993. Core-based trees (CBT): An Architecture for Scalable Inter-Domain Multicast Routing. In Communications, architectures, protocols, and applications San Francisco, CA, USA.

4. S. Deering, D.L. Estrin, D.Farinacci, V. Jacobson, C.-G. Liu, and L. Wei 1996. The PIM Architecture for Wide-Area Multicast Routing. IEEE/ACM Transactions on Networking, 4(2):153-162.

5. J. Moy 1994. Multicast Routing Extensions for OSPF. Communications of the ACM7 37(8):61-66.

6. Sung-Ju Lee, -William Su, Julian Hsu, Mario Gerla, and Rajive Bagrodia 2000. A Performance Comparison Study of Ad Hoc Wireless Multicast Protocols. In IEEE IWOGOM 2000, pp:565-574.

7. Mingyan Liu, Rajesh R. Talpade, and Anthony McAuley, 1999. AMRoute: Ad hoc Multicast Routing Protocol. Technical Report 99, The Institute for Systems Research, University of Maryland.

8. C.W. Wu and Y.C. Tay 1999. AMRIS: A Multicast Protocol for Ad hoc Wireless Networks. In IEEE Military Communications Conference (MILGOM), Atlantic City, NJ, pp: 25-29.

9. C. Ho, K. Obraczka, G. Tsudik, and K. Viswanath, 1999. Flooding for Reliable Multicast in Multihop Ad Hoc Networks. In The 3rd Intl. workshop on Discrete Algorithms and Methods for Mobile Computing and Communications (DIAL-M'99).

10. Raghupathy Sivakumar, Bevan Das, and Vaduvur Bharghavan, 1998. Spine Routing in Ad hoc Networks. A CM/Baltzer Publications Cluster Computing Journal, Special Issue on Mobile Computing.

11. P. Sinha, R. Sivakumar, and Bi. Bharghavan, 1999. CEDAR: A Core- Extraction Distributed Ad hoc Routing Algorithm. In IEEE INFOCOM' 99, New York City, NY.

12. Ulas C. Kozat, George Kondylis, Bo Ryu, and Mahesh K. Marina, 2001. Virtual Dynamic Backbone for Mobile Ad Hoc Ketworks. In IEEE International Conference on Communications (ICC), Helsinki, Finland. 